\title{
Self-Recognition and Other-Recognition in Point-Light Displays
}

\author{
Robert W. Mitchell*, Crystal Curry \\ Department of Psychology, Eastern Kentucky University, Richmond, KY, USA

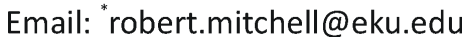

Received 4 October 2015; accepted 15 February 2016; published 18 February 2016

Copyright (C) 2016 by authors and Scientific Research Publishing Inc.

This work is licensed under the Creative Commons Attribution International License (CC BY). http://creativecommons.org/licenses/by/4.0/

c) (i) Open Access

\begin{abstract}
Adult humans' recognition of self and others in diverse media (e.g., mirrors, videos) provides evidence for their skills at kinesthetic-visual and visual-visual matching, respectively. In this study, we examine self- and other-recognition in point-light displays (PLDs). Participants (7 men, 4 women) were filmed while walking in the dark with lighted joints to create two PLDs each, one showing a frontal view and one showing a half-profile view. Ten of the participants then observed 22 PLDs, two of themselves and two each of 10 familiar persons, and named whom they perceived in each PLD. Participants achieved greater than chance levels of accuracy in identifying themselves (55\% of the time) and others (29.5\%). Comparisons using three measures showed that participants were better at detecting themselves than others; however, variability in self- and other-detection within and across studies suggests caution prior to generalizing. Participants were equally successful in detecting walkers in frontal and half-profile PLDs, on average detecting about 3 walkers out of 11 in each perspective. Thus, participants showed some skill in using kinesthetic-visual and visual-visual matching in recognizing self and other, respectively, from the limited information present in PLDs.
\end{abstract}

\section{Keywords}

Self-Recognition, Other-Recognition, Point-Light Display, Kinesthetic-Visual Matching, Visual-Visual Matching

\section{Introduction}

Awareness that the form or style of one's bodily movements is being replicated, a type of self-recognition, is the result of developing skill at matching between kinesthetic experiences or memories and similar visual expe-

\footnotetext{
${ }^{*}$ Corresponding author.
} 
riences or images, i.e., "kinesthetic-visual matching” (Mitchell, 1993; 2012). Such kinesthetic-visual matching involves understanding that one can "mirror" another, or that one is being "mirrored," and requires a common code for comparison between perceptual modalities. To understand kinesthetic-visual matching, imagine that you see a man perform an odd gesture with his arm; if you close your eyes, you can act out that gesture because you can match between your visual experience of the man's gesture and your kinesthetic experiences ("motor cues”-Sevdalis \& Keller, 2009: p. 501) of moving your arm in the same way. Kinesthetic-visual matching is present upon initial awareness, usually in childhood, that one can and is seeing oneself in a mirror, but also in noticing that one is being imitated by someone and in detecting one's actions in a point-light display (PLD) or other recorded medium (e.g., video) (Beardsworth \& Buckner, 1981; Mitchell, 1993). Recognition of others in similar contexts (mirrors, imitation, PLDs, other media) is usually based on a matching between two visual images, or between a visual image and visual memories (via common perceptual codes), that may or may not require the "motor" component present in kinesthesis (Mitchell, 1993). Human skills at multisensory matching are highly sophisticated, likely the most sophisticated of all primates (Mitchell, 1994; Mitchell \& Gallaher, 2001; Sevdalis \& Keller, 2011). In this study, we examine participants' skill at detecting themselves and familiar others when observing PLDs of these people walking.

Adults as well as infants recognize PLDs as images of moving bodies (Das et al., 2009; Fox \& McDaniel, 1982; Johansson, 1973; Moore et al., 2006), and infants distinguish self from others in PLDs based on kinesthetic-visual contingency by 5 months of age (Schmuckler \& Fairhall, 2001). Self-recognition occurs later, however, and children develop recognition of others in mirrors and videos prior to self-recognition in these media (by around 18 - 24 months in Western cultures), and imitate others' actions and recognize others' actions being imitated prior to recognizing being imitated themselves (for review, see Mitchell, 2012).

Information available for kinesthetic-visual or visual-visual matching is quite limited in a PLD, such that perfect success in detection is unlikely. Although one might expect that matching within a modality (vision) is easier than matching across modalities (vision and kinesthesis), data from PLDs are equivocal, leaning toward selfrecognition being easier for adults. One PLD study (Cutting \& Kozlowski, 1977) had 7 friends, 6 of whom produced 10 PLDs each walking back and forth in profile, name themselves or specific others in the PLDs; they detected themselves on average 41.3\%, and others, on average 37.3\% (a nonsignificant difference). A replication study (Beardsworth \& Buckner, 1981) had 6 participants, all of whom knew and frequently observed each other over the previous 3 years or longer, name the walker in 10 PLDs of each person in profile. Participants correctly selected, on average, themselves $58.3 \%$, and others $31.6 \%$ (significantly different at $p<0.10$ ).

An elaboration on these studies examined participants' skill at detecting themselves and 11 other familiar people (using two groups, comprising co-workers known to each other for at least 6 weeks) from PLDs in three orientations: frontal view, half-profile view, and profile view (Jokisch, Daum, \& Troje, 2006). Participants identified themselves and others at greater than chance levels for all views, showing comparable levels of skill for both self and other in the frontal and half-profile views (26.6\% - 30\% success), but better skill at detecting themselves (30\%) than others (18.4\%) in the profile view.

Various studies have extended the range of actions but simplified the number of options for identifying the PLDs, usually to choosing either the self or a familiar and/or unfamiliar other. Participants identified PLDs of dancing, walking, and clapping as either themselves or another unfamiliar person with or without musical accompaniment (Sevdalis \& Keller, 2009; 2010); of their own or two unfamiliar others’ musical conducting (with or without the appropriate music) and walking (Wöllner, 2012); of themselves, a friend, or a stranger engaging in ten diverse actions (including walking) (Loula, Prasad, Harber \& Shiffrar, 2005); and of more and less familiar persons walking normally or at fast or slow paces (Jacobs, Pinto \& Shiffrar, 2004). These studies show generally that, with few options of persons to detect, people are better at detecting themselves than either familiar or unfamiliar persons, and at detecting that someone is familiar than that someone is a stranger; that they are better at detecting people when they display more dynamic bodily actions (e.g., dancing, conducting, boxing, as opposed to walking, clapping, laughing) and when their actions are in real time rather than fast or slow; and that accompanying music does not influence detection skill. Inclusion of strangers likely changes how participants approach the task: when known individuals are used, participants are asking themselves "Who does that look like?”; when strangers are included, participants are asked to notice whether they do or do not know who produced the PLD in the midst of self- and other-identification. Although detection of self (as opposed to a friend or a stranger) from gait information was comparable to chance levels in Loula et al. (2005), when PLDs of partici- 
pants walking are presented without strangers, observers detect themselves and known others in PLDs of walking at greater than chance accuracy.

The current study examines the skill of participants whose walks are well known to each other to name the walker from PLDs of the participants walking in a half-profile and a frontal view. We predict that participants will identify their own and others' walks at greater than chance accuracy for both views, and will be equally successful at detecting their own and others' PLDs in both views.

\section{Methods}

\subsection{Participants}

The participants of this experiment were faculty at a regional university. The study included 11 participants: 7 men and 4 women. All subjects had worked for the university for at least three years, had seen each other quite frequently during the work week, and knew each other well by name. The study was IRB approved and participation was voluntary, and no incentive was given. All participants were told that this was a study about the perception of people's movement in PLDs.

One of the participants, the transcriber, was filmed walking and did not participate in the observation part of the study, as he created the study materials and thus became familiar with the PLDs of the walkers. Consequently, 11 people participated in the creation of the PLDs, but only 10 participants observed the PLDs to name the walkers. To avoid Type 2 error, we employed $\alpha=0.10$.

\subsection{Materials}

All eleven participants were individually filmed as point-light actors. Each participant wore his or her own clothing to which 13 yellow glow stick bracelets were attached to their major joints (ankles, knees, hips, wrists, elbows, shoulders, and a hat worn on their head). (Note that this number of lighted joint locations is about 1/3 of the number used in other studies, e.g. Jokisch et al., 2006.) Each end of the glow sticks was wrapped with black electrical tape so that only two and a half inches was visible. A 7-inch piece of black yarn was placed in the electrical tape at each end so that it could be wrapped around and attached to each joint. Each participant walked in a blackened room while being filmed with a digital camcorder. For the filming of the frontal view the camera was positioned at a fixed point in the room about $8 \mathrm{~m}$ away from the starting point of the participants, who walked toward the camera. For the filming of the half-profile view, the participants walked at a $45^{\circ}$ angle to the camera, from left to right, starting at a point to their right of where they began their walk with a frontal view. The tallest participant was used as a reference for height, and markers were placed at the foot and head on the camera itself to be used as reference points for all other participants. The camera's zoom button was used to fix each participant to the reference points so that the base of the feet and the top of the hat were always started at the same spot. Participants were asked to walk the room 3 - 4 times from a frontal view and 3 - 4 times from a half-profile view, for a total of 6 - 8 trips across the room. Participants were asked to walk as naturally as possible each time.

The digital camcorder recorded each participant onto a labeled mini-disc. Each mini disc was loaded and copied individually, using the program Nero 9, onto a laptop computer to create separate files. Each file was then converted and burned onto a DVD. One of the walkers (the "transcriber," who did not otherwise participate in the study) selected two PLDs from each walker, one from the frontal view and one from the half-profile view, as having the best visual display, and transferred these to one DVD for each walker. Each DVD contained two files: one 5 - 6 seconds of a frontal walk, and the other 5 - 6 seconds of a half-profile walk. The transcriber wrote an arbitrary symbol for each walker on the DVD containing his or her PLDs, so that the experimenter was blind to the identity of the person whose PLD was being shown.

Each participant viewed 11 frontal walk PLDs, and 11 half-profile walk PLDs. Each participant's sequence was unique. In creating the sequence of walks to be shown to each person, the transcriber alternated frontal view and half-profile view PLDs. In addition, half of the participants began by viewing a frontal view PLD, and other half began by viewing a half-profile view PLD. The transcriber also varied the position of the observer's own walks in the sequence he or she observed, using the method shown in Table 1. Thus, 5 participants began with a PLD from the front (and two of these observed their own PLD first), and 5 began with a PLD with the half-profile (and two of these observed their own PLD first). Participants were randomly assigned to one of the 
Table 1. Arrangement of participant’s own appearance in the sequence of walks observed.

\begin{tabular}{cccc}
\hline \multirow{2}{*}{ Gender } & Began with & Self Appeared & Self Appeared \\
\cline { 3 - 4 } Male A & on Frontal Trial \# & 3 & 6 \\
Male B & Front & 3 & 6 \\
Male C & Front & 6 & 1 \\
Male D & Front & 1 & 9 \\
Male E & Side & 1 & 3 \\
Male F & Side & 9 & 1 \\
Female J & Front & 1 & 3 \\
Female K & Front & 9 & 1 \\
Female L & Side & 1 & 9 \\
Female M & Side & 6 & 1 \\
\hline
\end{tabular}

arrangements in Table 1, with the provision that an equal number of persons of each sex began with either a frontal view or a half-profile view. In order to give each person an equal chance of being selected at each point in the sequence of frontal and half-profile trials, the experimenter created an array for frontal trials, and another array for half-profile trails, such that for any given frontal trial or any given half-profile trial, each walker's PLD appeared only once across subjects. Thus, for frontal trial number 1, across sessions, each walker's frontal view PLD was presented only once, and the same for half-profile trail number 1, frontal trial number 2, half-profile trial number 2, etc. In this way, each participant received a different sequence of trials; if participants varied in their responses during the session, the overall percentage correct for each participant, and each participant's walk, would be unaffected.

The transcriber provided the sequence of PLDs to be shown to each participant using the symbols on the dvds, so that the experimenter simply matched the symbol in the sequence to the symbol on the dvd. The symbols were later matched to the walkers' names.

\subsection{Procedure}

A week after filming, ten participants were invited back individually to complete the experiment. The experiment consisted of a choice task of identification in which each participant attempted to identify the actors in the PLDs. During the experiment, the participants were seated in frontal of a Lenovo laptop computer in a dimly light room. They were given pen, paper, and the list of names of the walkers so that they could write the name of the walker they perceived to be moving in the PLD. They were also provided with an easy sudoku to do between observations. Participants were told that everyone on the list was featured at least once in the sequence of PLDs, but that a person could appear more than once. They were also told that a PLD would be played on repeat until they wrote a response on the sheet of paper. Participants' selections were correct when they provided the name of the walker they had observed, and incorrect otherwise. Once they made a response they were to work on the sudoku until discs had been changed (which took about 30 - 45 seconds). The PLD segments were played in this fashion until all 22 PLDs had been shown. Participants received no feedback about their selections. Each session took less than half an hour to complete.

\section{Results}

\subsection{Detection of Target}

On average, participants detected the target 7 out of 22 times $(S D=3.23)$. Assuming that the probability of detecting the target each time was $1 / 11$, the probability of detecting 7 or more targets out of 22 opportunities by chance is unlikely (Binomial, $N=22, k=7, p<0.003$ ).

\subsection{Selections of Individuals as Walkers}

Each person was selected a minimum of 0 times and a maximum of 5 times by viewers (see Table 2). Individuals were selected, on average, 20 times across observers. Some individuals were selected as the walkers more 
Table 2. Minimum and maximum number of times observer picked any given name, number of names selected, and average percent correct per name selected.

\begin{tabular}{|c|c|c|c|c|}
\hline \multirow{2}{*}{ Observer } & \multirow{2}{*}{ Minimum } & \multirow{2}{*}{ Maximum } & No. names & \multirow{2}{*}{$\begin{array}{c}\text { Mean \% correct } \\
\text { per name }\end{array}$} \\
\hline & & & selected & \\
\hline M & 1 & 5 & 11 & 0.145 \\
\hline $\mathrm{D}$ & 0 & 4 & 10 & 0.225 \\
\hline E & 1 & 4 & 11 & 0.258 \\
\hline K & 1 & 3 & 11 & 0.227 \\
\hline $\mathrm{F}$ & 1 & 3 & 11 & 0.227 \\
\hline $\mathrm{C}$ & 1 & 3 & 11 & 0.242 \\
\hline A & 0 & 3 & 10 & 0.283 \\
\hline $\mathrm{L}$ & 1 & 3 & 11 & 0.333 \\
\hline $\mathrm{J}$ & 1 & 3 & 11 & 0.515 \\
\hline B & 1 & 3 & 11 & 0.667 \\
\hline
\end{tabular}

than others. Excluding participants' selections of themselves, names of individual walkers were selected a total of 14 - 28 times across observers; including these selections, participants were selected from 16 - 28 times.

One could argue that participants were more likely to obtain success in correctly detecting the walker by more frequent selection of individuals. However, any increased selection for one individual increased the number of errors, such that this strategy would not be (and was not—see Table 2) effective overall.

It may be the case that some people had walks that were distinctive or particularly recognizable in some way. The frequency of correct selections of each walker is presented in Table 3. The walker detected the most frequently by others oddly failed to detect himself; the walker with the next most frequent detection rate detected herself both times. To determine whether any particular selection was especially frequent, a z distribution was created for the number correct for each walker, where $M=6.36$ and $S D=3.11$. Only the most frequently detected walker's frequency of detection was greater than two standard deviations away from the mean, suggesting that one of the walkers may have been more distinctive than the others (though, again, not to himself).

\subsection{Detection of Self}

Each participant was shown 2 PLDs of him/herself, one each from the frontal and half-profile views. The probability of participants correctly detecting themselves by chance in one or both PLDs is 0.1736 . (The probability of incorrectly selecting the self 2 times is [10/11] $\times$ [10/11], or 0.8264 . The probability of correctly selecting the self at least once [i.e., once or twice] is, then, 1 - 0.8264, or 0.1736.) Participants correctly selected themselves, on average, 1.1 times $(S D=0.738$ ) out of the 2 times that they were shown PLDs of themselves (i.e., $55 \%$ of the time), which is a greater than chance likelihood of detection, $t(9)=3.97, p<0.01, r^{2}=0.64$ (see Table 3 and Table 4). Eight out of 10 participants detected themselves at least once, which is more likely than expected by chance: Binomial test, $N=10, k=8, p<0.001$. In fact, three of them detected themselves both times they observed themselves, which is unlikely by chance, given a chance probability of $(1 / 11) \times(1 / 11)=0.0083$ each time: Binomial test, $N=10, k=3, p<0.001$. For each participant, the probability of detecting themselves by chance in the frontal view, or in the half-profile view, is $1 / 11$ or 0.0909 . In the frontal view of themselves, 4/10 participants detected themselves; in the half-profile view of themselves, 7/10 participants detected themselves. The probability of 4 or more participants correctly detecting themselves by chance in either case is unlikely (Binomial test, $N=10, k=4, p<0.01$ ). Thus, it appears that more participants detected themselves at least once from either view than chance would predict ${ }^{1}$.

Participants selected themselves (correctly and incorrectly) as the walker in PLDs, on average, 1.9 times (SD = 0.88 ), which was statistically indistinguishable from a random selection of themselves 2 times, $t(9)=-0.36, p=$ 0.73. The number of times participants selected themselves did not affect their accuracy. One person selected

\footnotetext{
${ }^{1}$ The placement of the participant's own PLDs in the sequence he or she observed seems to have had little effect on detection: accurate detection of self occurred for 3 of 4 participants whose PLD was on their first trial; 1 of 4 , on the second trial; 2 of 4 , on the fifth or sixth trial; 3 of 4 , on the 11th or 12th trial; and 2 of 4 , on the 17th or 18th trial.
} 
Table 3. The frequency with which particular walkers were correctly selected by self and others.

\begin{tabular}{cccc}
\hline Walker & Frequency & Self & Others \\
\hline D & 2 & 1 & 1 \\
J & 3 & 0 & 3 \\
L & 4 & 1 & 4 \\
A & 5 & 1 & 4 \\
B & 6 & 2 & 6 \\
G & 6 & - & 5 \\
C & 7 & 2 & 6 \\
F & 7 & 1 & 6 \\
M & 7 & 1 & 8 \\
K & 10 & 2 & 13 \\
E & 13 & 0 & 59 \\
Totals & 70 & 11 & \\
\hline
\end{tabular}

Table 4. Number of selections of correct detections of self PLDs, non-self PLDs, and non-self participants at least once, as well as total number of correct detections.

\begin{tabular}{ccccc}
\hline & \#Correct & \#Correct & \#Non-self walkers for whom & Total \\
\cline { 2 - 5 } Observer & Self & Non-self & At least 1 of 2 selections is correct & \#Correct \\
\hline M & 1 & 3 & 2 & 4 \\
K & 2 & 4 & 3 & 5 \\
E & 0 & 5 & 4 & 6 \\
D & 1 & 5 & 4 & 6 \\
C & 2 & 4 & 4 & 6 \\
A & 1 & 5 & 4 & 5 \\
F & 1 & 4 & 6 & 7 \\
L & 1 & 6 & 8 & 10 \\
J & 0 & 10 & 9 & 15 \\
B & 2 & 13 & 48 & 70 \\
Totals & 11 & 59 & 4 & \\
\hline
\end{tabular}

himself 4 times, and was correct only once. Six participants selected themselves twice; half were correct both times, half were correct only once. Three participants selected themselves once; one was correct, the other two were not.

\subsection{Detection of Others}

Each participant was shown 20 walks of others, one each from the frontal and half-profile views of the 10 other participants. Participants correctly named, on average, 5.9 walkers $(S D=3.14)$ on the 20 trials concerning walkers other than themselves (i.e., $29.5 \%$ of the time). The median was 5 correct out of 20 . One man correctly named 13 PLDs of others, one woman correctly named 10, and the rest correctly named between 3 and 6 (see Table 4).

An observing participant might have correctly selected one participant both times they appeared, another only once in the two times he or she appeared, and another neither time he or she appeared. So the selections of the other can be evaluated for each participant observed as either zero correct, or at least one of the two times correct. The probability of correctly selecting the appropriate participant for one or both of these views, for each participant observed, is 0.1736 , so the expected frequency of correct selections by chance of one or both views for each observing participant across the 10 pairs of observations is 1.736 correct selections. For the 10 partici- 
pants, the average number of pairs of trials (out of 11) for each observer in which at least one selection was correct is $4.8(S D=2.20)$; based on a one-sample t-test, this is significantly different from chance: $t(9)=4.40, p<$ $0.01, r^{2}=0.68$. In general, observing participants detected other participants at least once in each pair of observations for the 10 other participants at greater than chance levels.

Each observer watched 10 PLDs of others from the frontal view, and 10 PLDs of others from the half-profile view. The probability of correctly selecting the appropriate participant by chance for each frontal view, or each half-profile view, is $1 / 11$, or 0.0909 . The expected frequency of correct selections for each participant across the 10 frontal view trials (as well as across the 10 half-profile view trials) is 0.90909 . For the 10 frontal views, $M=$ $3.2(S D=2.15)$; using a one-sample t-test, $t(9)=4.57, p<0.01, r^{2}=0.70$. For the 10 half-profile views, $M=2.7$ $(S D=1.49)$; using a one-sample t-test, $t(9)=5.52, p<0.01, r^{2}=0.77$. Thus, it appears that more participants detected other participants from the frontal and/or half-profile views than chance would predict.

\subsection{Comparison between Detection of Self and Others}

On average, participants detected themselves 1.1 times per 2 observations (55\% of the time), and detected others 5.9 times per 20 observations (29.5\% of the time) (see Table 4.). It is difficult to compare these two measures statistically, given the differences in the probabilities of success for each set of walkers (for self-detection, only $0,0.5$, and 1.0; for detection of others, the range is from 0.15 to 0.65 ). Thus, to examine all reasonable comparisons between detection of self and other, the percent correct score (out of 2 trials) for detecting the self was compared, separately, to three measures of the percent correct for the other: the absolute percent correct (out of 20 trials) by the same participant for detecting any others, $t(9)=2.05, p=0.07, r^{2}=0.32$; the percent correct using the median (or-identical_-modal) number correct out of 2 trials by the same participant for detecting any others across nonself participants, $t(9)=2.75, p=0.022, r^{2}=0.46$; and the percent correct (out of 20 trials) for the detection of the participant by all others, $t(9)=2.18, p=0.058, r^{2}=0.35$. (The same pattern of results occurred when the data were subjected to Wilcoxon tests.) All comparisons indicated that self-recognition was better than other-recognition, and that who was being detected (self or other) explained 32\% - 46\% of the variance in recognition-a large effect size.

Across all participants, detecting oneself occurred more frequently (or more easily) than detecting most other participants. Participants detected themselves 11 times out of 20; one walker was detected by others 13 times out of 20, but the rest were detected by others 8 or fewer times (see Table 4). To determine if observers' variation in skill in detecting themselves was related to their PLDs being somehow more or less easily detectable by others, we correlated the number of times observers correctly identified their own PLDs with the number of times these observers' PLDs were correctly detected by others. The correlation was not significant: $r=-0.194, p=0.60$. Thus, visual distinctiveness of individuals' PLDs was unrelated to their detection of themselves, as would be expected from different processes being used to recognize self and other: kinesthetic-visual matching to detect the self, and visual-visual matching to detect the other.

\subsection{Frontal versus Half-Profile Views}

Names of 34 PLDs were correctly detected from walks with a frontal view, and 36, from walks with a half-profile view. One walker was detected 6 times only from the frontal view; one walker was detected twice, and one, 3 times, only from the half-profile view. The other 8 walkers were detected from both sides across participants. The 10 observers correctly detected the same number of walkers from the frontal $(M=3.60$ [i.e., $32.7 \%$ ], $S D=2.22)$ and half-profile views $(M=3.40$ [i.e., $30.9 \%$ ], $S D=1.51 ; t(9)=0.32, p=0.76$; if self images are excluded, the observers also correctly detected the same number of walkers (other than themselves) from the frontal $(M=3.20$ [i.e., $32 \%$ ], $S D=2.15)$ and half-profile views $(M=2.70$ [i.e., $27 \%$ ], $S D=1.49 ; t(9)=$ $0.80, p=0.44$. The self was detected no better from the frontal (40\%) or half-profile (70\%) view: exact McNemar test, $p=0.375$. The 11 walkers were correctly detected with the same frequency, on average, from the frontal $(M=3.27$ [i.e., 29.7\%], $S D=1.95)$ and half-profile views $(M=3.09$ [i.e., 28.1\%], $S D=2.34) ; t(10)=0.20$, $p=0.84$. Apparently neither view per se afforded better detection.

\section{Discussion}

Participants detected both self and others at greater than chance frequencies, providing evidence of some skill at 
kinesthetic-visual and visual-visual matching of images of bodies, consistent with findings in previous research using similar methods (Beardsworth \& Buckner, 1981; Cutting \& Kozlowski, 1977; Jokisch, Daum, \& Troje, 2006). However, these data conflict with those of Loula et al. (2005), whose participants showed no skill at detecting their own or another's walk. This discrepancy is not surprising, as the task in the Loula et al. study was different than in earlier studies and our own, where participants were required to attend to stylistic characteristics in movement in the PLDs to detect individuals. By contrast, the Loula et al. study required use of disparate skills: participants could select themselves, a friend, or a stranger as the walker, such that attention to walking style was unavailable for the stranger PLDs. Instead of trying to detect individuals, the participants in Loula et al. had to decide between known and unknown movements styles, and if known, between their own and another's.

The greater success at self-recognition than other-recognition for PLDs suggests that cross-modality matching between degraded visual images of oneself and one's kinesthetic memory of one's own movements is usually easier than within-modality matching between degraded visual images of others and one's visual memory of them. However, variability across participants in skill at detecting self and others is present both within and across studies (Beardsworth \& Buckner, 1981; Cutting \& Kozlowski, 1977; Jokisch, Daum, \& Troje, 2006). Detectability of self was better than that of others for frontal and half-profile views of the PLD walker in our study, whereas no such distinction was discerned by Jokisch, Daum, and Troje (2006). The difference between these studies is not in the detection of others: both studies showed almost identical other-detection rates (frontal view: theirs: $28.6 \%$, ours: $32 \%$; half-profile view: theirs: $26.6 \%$, ours $27 \%$ ). Rather, the difference is in self- detection rates: whereas self-detection in their study was $27.3 \%$ for the frontal and $30 \%$ for the half-profile view, self-detection in our study was $40 \%$ for the frontal and $70 \%$ for the half-profile view. One might suspect from these discrepancies across studies that differences and similarities between self- and other-detection rates occur specifically because of variability in self-detection, but in fact measures of other-detection are also variable across studies: other-detection rates for profile view PLDs were 37.3\% for Cutting and Kozlowski (1977) and 31.6\% for Beardsworth and Buckner (1981), but only 18.4\% for Jokisch, Daum, and Troje (2006). Thus, the claim by Jokisch, Daum, and Troje (2006) that detection of self is viewpoint independent (i.e., consistent across all three viewing conditions), but detection of others is viewpoint dependent (i.e., inconsistent), requires replication, as the discrepancy they detected may result from individual variability in self- and other-detection skills.

Future work might usefully look to explain why some people are better at self-detection, and others, at other-detection. Perhaps some measures of personality, such as independent vs. interdependent self-construal (Markus \& Kitayama, 1991), could usefully predict success on self- and other-detection in PLDs. Reaction times might also be used as a measure of ease or difficulty in detection of self and others. In addition, which aspects of style in walking are detected in PLDs could be examined through self-report. Participants may be aware of salient aspects of individuals' walking style that allow them to identify PLDs. In our study, some walkers appeared to be more detectable than others, with one walker whom all participants (except for two, one of whom was himself) detected at least once, and half of those detected twice. By contrast, some walkers seemed hard to detect: one walker was detected only once by himself, and once by another.

PLDs provide extensive though imprecise visual information about the self and others. Given that we rarely see ourselves move, our PLD self-recognition must rely on kinesthetic-visual matching (Beardsworth \& Buckner, 1981; Mitchell, 1993; 2012), which matching obviously relies on a common code across perceptual modalities (Prinz, 1997) that allows for such comparison. Style of walking is, of course, only one of many pieces of information that we usually use to identify (aspects of) ourselves and others, so the high frequency of misidentifications of PLDs is not unexpected.

\section{Acknowledgements}

We greatly appreciate reviewers' comments, which helped us to improve the paper.

\section{References}

Beardsworth, T. \& Buckner, T. (1981). The Ability to Recognize Oneself from a Video Recoding of One’s Movements without Seeing One's Body. Bulletin of the Psychonomic Society, 18, 19-22. http://dx.doi.org/10.3758/BF03333558

Cutting, J. E., \& Kozlowski, L. T. (1977). Recognizing Friends by Their Walk: Gait Perception without Familiarity Cues. Bulletin of the Psychonomic Society, 9, 353-356. http://dx.doi.org/10.3758/BF03337021

Das, S. R., Lazarewicz, M. T., Wilson, R. C., \& Finkel, L. H. (2009). Sensitivity to Motion Features in Point Light Displays 
of Biological Motion. Spatial Vision, 22, 105-125. http://dx.doi.org/10.1163/156856809787465627

Fox, R., \& McDaniel, C. (1982). The Perception of Biological Motion by Human Infants. Science, 218, 486-487. http://dx.doi.org/10.1126/science.7123249

Jacobs, A., Pinto. J., \& Shiffrar, M. (2004). Experience, Context, and the Visual Perception of Human Movement. Journal of Experimental Psychology: Human Perception and Performance, 30, 822-835. http://dx.doi.org/10.1037/0096-1523.30.5.822

Johansson, G. (1973). Visual Perception of Biological Motion and a Model for Its Analysis. Perception \& Psychophysics, 14, 201-211. http://dx.doi.org/10.3758/BF03212378

Jokisch, D., Daum, I., \& Troje, N. F. (2006). Self Recognition versus Recognition of Others by Biological Motion: Viewpoint-Dependent Effects. Perception, 35, 911-920. http://dx.doi.org/10.1068/p5540

Loula, F., Prasad, S., Harber, K., \& Shiffrar, M. (2005). Recognizing People from Their Movement. Journal of Experimental Psychology: Human Perception and Performance, 31, 210-220. http://dx.doi.org/10.1037/0096-1523.31.1.210

Markus, H., \& Kitayama, S. (1991). Culture and Self: Implications for Cognition, Emotion, and Motivation. Psychological Review, 98, 224-253. http://dx.doi.org/10.1037/0033-295X.98.2.224

Mitchell, R. W. (1993). Mental Models of Mirror-Self-Recognition: Two Theories. New Ideas in Psychology, 11, $295-325$. http://dx.doi.org/10.1016/0732-118X(93)90002-U

Mitchell, R. W. (1994). The Evolution of Primate Cognition: Simulation, Self-Knowledge, and Knowledge of Other Minds. In D. Quiatt, \& J. Itani (Eds.), Hominid Culture in Primate Perspective (pp. 177-232). Boulder: University Press of Colorado.

Mitchell, R. W. (2012). Self-Recognition in Animals. In M. R. Leary, \& J. P. Tangney (Eds.), Handbook of Self and Identity (2nd ed., pp. 656-679). New York: Guilford.

Mitchell, R. W., \& Gallaher, M. (2001). Embodying Music: Matching Music and Dance in Memory. Music Perception, 19, 65-85. http://dx.doi.org/10.1525/mp.2001.19.1.65

Moore, D. G., Goodwin, J. E., George, R., Axelsson, E. L., \& Braddick, F. M. B. (2006). Infants Perceive Human PointLight Displays as Solid Forms. Cognition, 104, 377-396. http://dx.doi.org/10.1016/j.cognition.2006.07.007

Prinz, W. (1997). Perception and Action Planning. European Journal of Cognitive Psychology, 9, 129-154. http://dx.doi.org/10.1080/713752551

Schmuckler, M. A., \& Fairhall, J. L. (2001). Visual-Proprioceptive Intermodal Perception Using Point Light Displays. Child Development, 72, 949-962. http://dx.doi.org/10.1111/1467-8624.00327

Sevdalis, V., \& Keller, P. E. (2009). Self-Recognition in the Perception of Actions Performed in Synchrony with Music. Annals of the New York Academy of Sciences, 1169, 499-502. http://dx.doi.org/10.1111/j.1749-6632.2009.04773.x

Sevdalis, V., \& Keller, P. E. (2010). Cues for Self-Recognition in Point-Light Displays of Actions Performed in Synchrony with Music. Consciousness and Cognition, 19, 617-626. http://dx.doi.org/10.1016/j.concog.2010.03.017

Sevdalis, V., \& Keller, P. E. (2011). Captured by Motion: Dance, Action Understanding, and Social Cognition. Brain and Cognition, 77, 231-236. http://dx.doi.org/10.1016/j.bandc.2011.08.005

Wöllner, C. (2012). Self-Recognition of Highly Skilled Actions: A Study of Orchestral Conductors. Consciousness and Cognition, 21, 1311-1321. http://dx.doi.org/10.1016/j.concog.2012.06.006 